\title{
High temperature in situ SEM assessment followed by ex situ AFM and EBSD investigation of the nucleation and early growth stages of Fe-Al intermetallics
}

\author{
T. Sapanathan ${ }^{1, *}$, I. Sabirov², P. Xia ${ }^{2,3}$, M.A. Monclús ${ }^{2}$, J.M. Molina-Aldareguía ${ }^{2}$, P.J. Jacques ${ }^{1}$, A. Simar ${ }^{1}$ \\ ${ }^{1}$ UCLouvain, Institute of Mechanics, Materials and Civil Engineering, IMAP, B-1348 Louvain-la-Neuve, \\ Belgium \\ ${ }^{2}$ IMDEA Materials Institute, Calle Eric Kandel 2, 28906 Getafe, Madrid, Spain \\ ${ }^{3}$ Universidad Politécnica de Madrid, E.T.S. de Ingenieros de Caminos, 28040 Madrid, Spain \\ * Corresponding author e-mail: thaneshan.sapanathan@uclouvain.be
}

\begin{abstract}
A dedicated in situ heating setup in a scanning electron microscope (SEM) followed by an ex situ atomic force microscopy (AFM) and electron backscatter diffraction (EBSD) is used to characterize the nucleation and early growth stages of Fe-Al intermetallics (IMs) at $596^{\circ} \mathrm{C}$. A location tracking is used to interpret further characterization. Ex situ AFM observations reveal a slight shrinkage and out of plane protrusion of the IM at the onset of IM nucleation followed by directional growth. The formed interfacial IM compounds were identified by ex situ EBSD. It is now clearly demonstrated that the $\theta$ phase nucleates first prior to the diffusion-controlled growth of the $\eta$-phase. The $\theta$-phase prevails the intermetallic layer.
\end{abstract}

Keywords: intermetallic compounds, interface diffusion, phase transformation, in situ heat treatments, electron backscatter diffraction (EBSD) 
Different Fe-Al intermetallics (IMs), also known as iron aluminides, can form by reaction implying diffusion in the Fe-Al binary system. The exact reaction scheme and the observed coexistence of several IMs when considering the reactions occurring at an interface between Fe and Al still need to be unveiled. Specific attention has been paid in literature to $\mathrm{Fe}_{3} \mathrm{Al}-\beta 1(<50$ at.\% Al), which offers good resistance to sulfidation, oxidation at high temperature and mechanical wear, while presenting a relatively high ductility among all the Fe/Al IMs and a lower density than steel [1]. As summarized in Table 1, the other Al-rich ( $\geq 50$ at.\% Al) Fe-Al IMs are FeAl $-\beta 2, \mathrm{FeAl}_{2}-\zeta, \mathrm{Fe}_{2} \mathrm{Al}_{5}-\eta, \mathrm{Fe}_{4} \mathrm{Al}_{13}-\theta$ (also referred to as $\mathrm{FeAl}_{3}$ in some early literature) and $\mathrm{Fe}_{5} \mathrm{Al}_{8}-\varepsilon$ (only stable at temperatures above $1095{ }^{\circ} \mathrm{C}$ [2]). The $\beta 2$ and $\zeta$ phases form via decomposition of the $\varepsilon$-phase resulting in a lamellar microstructure [3-5]. $\beta 2$ and $\zeta$ have thus also gained some attention for providing improved mechanical properties similar to the more commonly known TiAl-based alloys [5]. The other two Al-rich IMs, n- and $\theta$ - phases, have very low reported fracture toughness of $0.82 \mathrm{MPa} \cdot \mathrm{m}^{1 / 2}$ and $0.97 \mathrm{MPa} \cdot \mathrm{m}^{1 / 2}$, respectively [6]. Such low toughness IMs are undesirable for many engineering applications.

It is well established that the brittleness of the IM phases increases with increasing at.\% Al content. However, the Al-rich IMs (especially the $\eta$ - and $\theta$ - phases) spontaneously form during various manufacturing processes, e.g. welding, coating, sintering, aluminum casting with ferrous inserts, hotdip aluminizing, etc. [7-10]. Therefore, understanding the formation mechanism of the $\eta$ - and $\theta$ phases is crucial to avoid their undesirable formation during manufacturing processes. During these processes, the local temperature at the interface rapidly changes with time, which could play a significant role on the sequence of IM phase nucleation.

Table 1: Lattice structure, stability range, standard enthalpy and entropy change at $298 \mathrm{~K}$ and Gibb's free energy changes at $973 \mathrm{~K}$ for IMs of the Fe-Al binary system [5, 11-13]*

\begin{tabular}{llllll}
\hline $\begin{array}{l}\text { Intermetallic } \\
\text { phases }\end{array}$ & Lattice structure & $\begin{array}{l}\text { Stoichiometry } \\
\text { of Al (at.\%) }\end{array}$ & $\begin{array}{l}\Delta H_{298} \\
\left(\mathrm{~J} \mathrm{~mol}^{-1}\right)\end{array}$ & $\begin{array}{l}\Delta S_{298} \\
\left(\mathrm{~K}^{-1} \mathrm{~mol}^{-1}\right)\end{array}$ & $\begin{array}{l}\Delta G_{973} \\
\left(\mathrm{~J} \mathrm{~mol}^{-1}\right)\end{array}$ \\
\hline $\mathrm{Fe} \mathrm{Al}(\beta 1)$ & DO3 & $23-34$ & -57372 & 28 & -4827 \\
$\mathrm{FeAl}(\beta 2)$ & $\mathrm{BCC}$ (order B2) & $23-55$ & -51240 & 51 & -11090 \\
$\mathrm{FeAl}_{2}(\zeta)$ & Triclinic & $66-66.9$ & -81900 & 73.3 & -16999 \\
$\mathrm{Fe}_{2} \mathrm{Al}_{5}(\eta)$ & Orthorhombic & $70-73$ & -194040 & 166.7 & -19636 \\
$\mathrm{Fe}_{4} \mathrm{Al}_{13}(\theta)$ & Monoclinic & $74.5-76.5$ & -112560 & 95.6 & -22869 \\
\hline
\end{tabular}

* The stable phases at ambient temperature are listed in ascending at.\% of Al 
To date, many ex situ analyses have been performed to understand the formation of Fe-Al IMs using dedicated set-ups and post treatments [9, 13-16]. In most of these studies, solid iron or steel was dipped into liquid or semi-solid Al at different temperatures and for different times. Early work [9] mentioned that the $\eta$-phase is dominating, and its growth follows a parabolic law for temperatures ranging from $715^{\circ} \mathrm{C}$ to $944^{\circ} \mathrm{C}$. In another study [17], the Fe-Al intermetallic growth kinetics parameters were determined for temperatures ranging from $500^{\circ} \mathrm{C}$ to $1100^{\circ} \mathrm{C}$ based on hot dipping experiments. Al diffusion was considered as the main mechanism during the $\eta$-phase growth, while Fe diffusion was also suggested as an active mechanism based on the emergence of Kirkendall voids in the $\mathrm{Fe}_{2} \mathrm{Al}_{5}$ layer [17]. It should be noted that in such ex situ analyses, the onset of nucleation and the growth of various IMs could not be captured due to their extremely fast kinetics which requires a sophisticated experimental setup. Therefore, the early stages of IM nucleation and growth in the Fe-Al system remain mostly unexplored.

Recently, in situ high temperature experiments have become an important tool to elucidate the behaviour at moving interfaces. Austenite - ferrite moving interface during high temperature treatment was successfully characterized for steel using in situ EBSD analysis $[18,19]$. In situ X-ray absorption tomography was used to characterize the $\mathrm{IM}$ growth at $700{ }^{\circ} \mathrm{C}$ (i.e. after Al melting), allowing the observation of tongue-like growth of the $n$-phase [20]. Synchrotron X-ray radiography was also used to characterize the IM growth at the liquid Al/ solid Fe interface [21]. The authors reported the formation of the $n$-phase at $850{ }^{\circ} \mathrm{C}$, whereas a dotted $\theta$-phase and a needle-like $\theta$-phase formed during solidification [21]. The nature of the phases was confirmed by electron back scatter diffraction (EBSD) analysis of the heat-treated samples after significant growth. The early sequence of formation of the phases could thus not be accurately captured during this in situ X-ray radiography study as this technique did not allow to distinguish between the various Fe-Al phases nor track phases location. Owing to in situ X-ray diffraction (XRD) measurements, the formation of $\eta$ - and $\zeta$ - phases below the melting temperature of aluminum during a slow heating at a rate of $10 \mathrm{C} / \mathrm{min}$ was also emphasized [22]. Moreover, in situ thermal treatment in a transmission electron microscope (TEM) identified that 
a needle like $\theta$-phase formed during soaking at $350^{\circ} \mathrm{C}$ [23]. The TEM observations suggested that the $\theta$-phase forms first during IM formation at the Fe-Al interface, while other studies are still not conclusive [20-22]. This scenario may be due to the temporal/spatial resolutions of the in situ devices used in those experiments, as well as their thermal inertia.

The main objective of the present work is to shed more light on the early stages of IMs nucleation and growth in the Fe-Al system. A controlled in situ heat treatment within a SEM chamber was followed by detailed microstructural characterization of the IM sites. Roll bonding (RB) was used to prepare bonded Al-Fe joints following the procedure described in [24]. DP 600 steel and AA6061-T6 aluminum having the dimensions of $200 \times 80 \times 0.9 \mathrm{~mm}^{3}(\mathrm{~L} \times \mathrm{W} \times \mathrm{H})$ and $200 \times 80 \times 3 \mathrm{~mm}^{3}$, respectively, were stacked in the order of DP600 / AA6061-T6 / DP600 and wrapped in a steel foil to avoid relative sliding during the rolling process. The chemical compositions of the DP600 and AA6061-T6 are provided in Table S1 in Supplementary. The sample was subjected to four rolling passes to obtain a thickness reduction ratio of 50\%. $200 \mu \mathrm{m}$-thick specimens were then extracted from the transverse cross section of the Rollbonded (RB) sample for the in situ heating experiment. Such thin samples were used for the in situ SEM experiments to eliminate the effect of thermal gradients along the thickness of the sample during heating. The prepared interface is shown in Fig. 1a revealing a clean interface without any visible defects or any intermetallic compounds at the interface. In the selected zone, two micropores observed on the surface were used as position tracking markers during the in situ experiment (Fig. 1a) and for subsequent ex situ AFM and ex situ EBSD analyses.

To identify the activation temperature required for the nucleation and growth of IMs, differential scanning calorimetry (DSC) experiments were performed with a PERSEUS ${ }^{\circledR}$ STA 449 F1/F3 equipment. The $2 \times 2 \times 2 \mathrm{~mm}^{3}$ samples obtained from the RB specimen were used in this experiment. An identified peak corresponding to the intermetallics formation is normalized by the formed total intermetallic volume $\left(0.00138 \mathrm{~mm}^{3}\right)$ and given in Fig. $1 \mathrm{c}$. It is worth noting that, due to the presence of multiple IMs, the DSC results are not normalized to the unit mass. The DSC experiment showed that the temperature 
required to start forming the IM is about $577^{\circ} \mathrm{C}$. Initial trials of the in situ SEM experiments were thus carried out at different temperatures ranging from $570{ }^{\circ} \mathrm{C}$ to $600{ }^{\circ} \mathrm{C}$ with $10{ }^{\circ} \mathrm{C}$ increments (and a soaking time of $60 \mathrm{sec})$. From the in situ SEM experiments, it was identified that a temperature of 596 ${ }^{\circ} \mathrm{C}$ is required for the nucleation and growth of IM compounds, which can be recorded with a reasonable resolution (see the Supplementary video). The temperature difference from the DSC scan and the in situ SEM observation can be attributed to the location of the thermocouple. Indeed, the DSC sample was placed inside an $\mathrm{Al}_{2} \mathrm{O}_{3}$ crucible in a furnace made of SiC chamber, and the thermocouple was located below that crucible; whereas in the SEM in situ experiment, the thermocouple was touching the sample surface (see Fig. 1b).
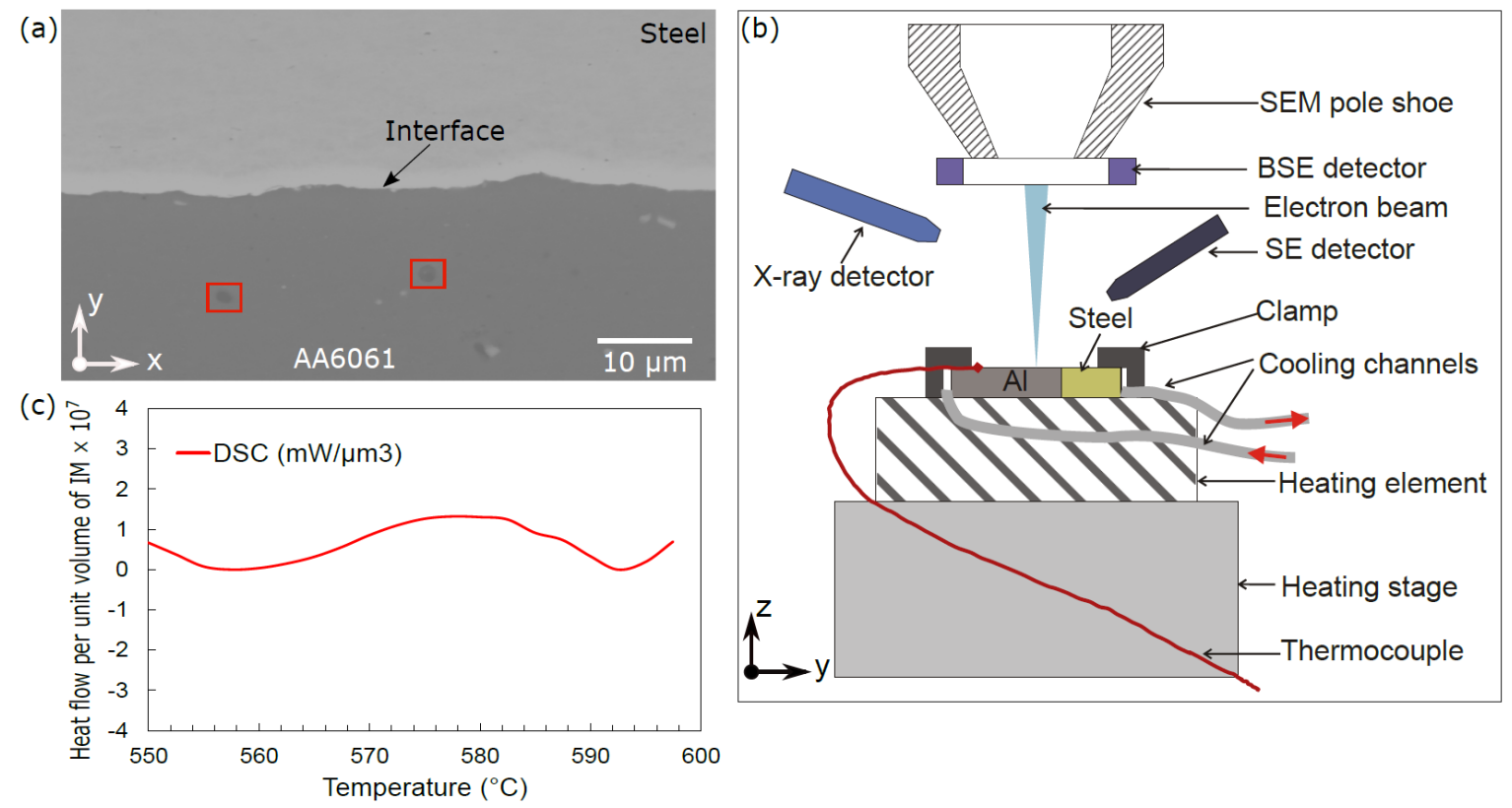

Fig. 1. (a) SEM micrograph of the interface of one RB sample used for the in situ experiments. The red squares indicate two micropores in the Al alloy that were used for position tracking during the in situ experiments and subsequent ex situ AFM and EBSD analysis. (b) Schematic illustration of the setup used for the high temperature in situ experiments inside the SEM chamber. (c) Heat flow behavior specific to the unit volume of intermetallic obtained from DSC measurement.

A ZEISS SEM EVO MA15 equipped with a heating stage, which is part of the Hysitron PI88 picoindenter, was used to carry out the in situ thermal treatment (Fig. 1b). Temperature was actively measured by a thermocouple placed in contact with the sample surface and feedback-controlled via a Lakeshore 336 PID temperature controller to ensure maximum thermal stability at the desired values (within \pm 
$0.01^{\circ} \mathrm{C}$ ). The temperature with time plot is given in the supplementary (Fig. S1). The microscope was operated at $18 \mathrm{kV}$. The electron beam scanned over the $69.2 \mu \mathrm{m} \times 51.9 \mu \mathrm{m}$ surface area perpendicular to the interface (y direction), and secondary electron (SE) signal was continuously recorded (at 12 frames/min) during heating. The pixel size of the SEM images was $67.4 \mathrm{~nm}$. This allowed to obtain SEM images of sufficient quality while keeping track of microstructure evolution during heating. After the heating stage, the topography of the nucleated IMs at the interface was characterized using an XE-150 Park's AFM system fitted with Acta ${ }^{\circledR}$ cantilever scanning tips. The AFM was operated in non-contact mode at a scan rate of $0.5 \mathrm{~Hz}$ to obtain topological images with typical scan areas of $10 \times 10 \mu \mathrm{m}^{2}$ at $512 \times 512$ pixels resolution. EBSD analysis of the same region (after fine polishing) was also performed using FEl Quanta TM Helios NanoLab 600i, equipped with a NordlysNano detector controlled by the AZtec Oxford Instruments Nanoanalysis software. The EBSD data were acquired at an accelerating voltage of $18 \mathrm{kV}$, a working distance of $8 \mathrm{~mm}$, a tilt angle of $70^{\circ}$ and a step size of $100 \mathrm{~nm}$ in a square scan grid mode.

Figures 2a-d illustrate the sequence of IM nucleation and growth during the in situ heating experiments. Image analysis was performed using the Image J software to quantitatively measure the extent of the IM growth at the interface (Fig. 2e). As shown in Fig. 2e, the IM nucleates at various locations during the first $10 \mathrm{~s}$ of soaking at $596{ }^{\circ} \mathrm{C}$, and a fast growth occurs during the first $40 \mathrm{~s}$ of soaking (for Locations B to F marked in Fig. 2d), followed by saturation of the growth. After the onset of nucleation (at $\mathrm{t}=0$ ), the initial IM growth rate is $\sim 0.183 \mu \mathrm{m} / \mathrm{s}$. However, when the growth is delayed by about $10 \mathrm{~s}$, the corresponding total IM growth rate at the beginning slowed down to $\sim 0.081 \mu \mathrm{m} / \mathrm{s}$ (indicated by the curve corresponding to Location A in Fig. 2e). It is worth noting that in Location A, the intermetallic still continues its growth at the end of the experiment $(60 \mathrm{~s})$, although at a lower rate. 

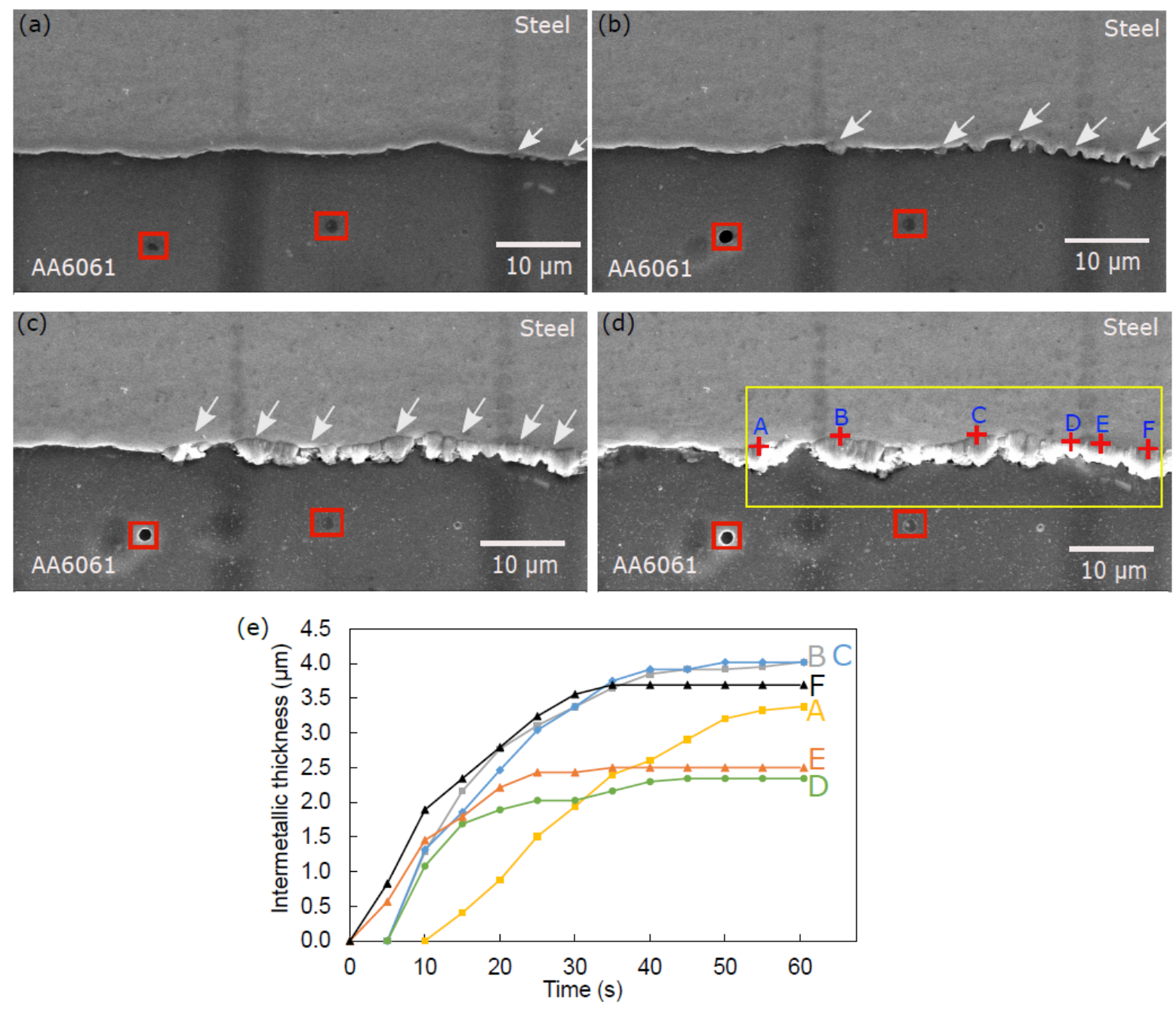

Fig. 2. Sequence of IM nucleation and growth obtained from in situ SEM observations at (a) $t=5 \mathrm{~s}$, where the onset of nucleation of IM is indicated by white arrows at the interface. The micropores marked by the red squares were used to track the position for further characterization (AFM and EBSD). (b) $t=10 \mathrm{~s}$; (c) $t=30 \mathrm{~s}$; (d) $t=60 \mathrm{~s}$, and (e) time dependent evolution of average IM thickness obtained using image analysis. Due to the low contrast difference between the different IMs formed during this observation and owing to the additional topological effect due to the 3D morphology of the newly formed IMs, the total IM thickness growth is plotted at various locations (marked by A-F in (d)) in (e). White arrows in Fig. 2a-c indicate the nucleation and growth of IMs. The yellow rectangle marked in Fig. $2 \mathrm{~d}$ refers to the zone corresponding to the EBSD analysis, discussed below in the manuscript. The complete recording of the IM nucleation and growth is provided as Supplementary Material.

After the in situ heating experiment, the 3D morphology of the nucleated IMs was characterized by ex situ AFM (Fig. 3) when the exact location was identified using red boxed micropores in Fig. 2. The AFM observations corresponding to locations A and B (Figs. 2d and e) show different growth behaviors. 3D views of the AFM maps are provided in Fig. S2 in the Supplementary Material. The AFM analysis revealed that the formation and growth of IMs were accompanied by local expansion/shrinkage. The 
IM compounds clearly pop up above the original surface of both Al and steel by up to $\sim 1.05 \mu \mathrm{m}$ due to the local expansion of the sample following solid state reaction [25], while some surrounding zones are clearly below the original surface (up to $-1 \mu \mathrm{m}$ ). This effect is more pronounced at location $A$, corresponding to an IM nucleation site (Fig. 3a), than at location B, corresponding to the significantly grown IM (Fig. 3b). It is clearly seen that at an early stage, the IM starts to pop up out of plane in a random direction (Fig. 3a). This may be attributed to the formation of the $\theta$-phase (as corroborated by further ex situ EBSD analysis), which nucleates first by reaction between Al and steel. The developed IM in Fig. 3b clearly shows a preferential growth perpendicular to the interface, which may be attributed to the diffusion-controlled growth of $\eta$-phase [26].
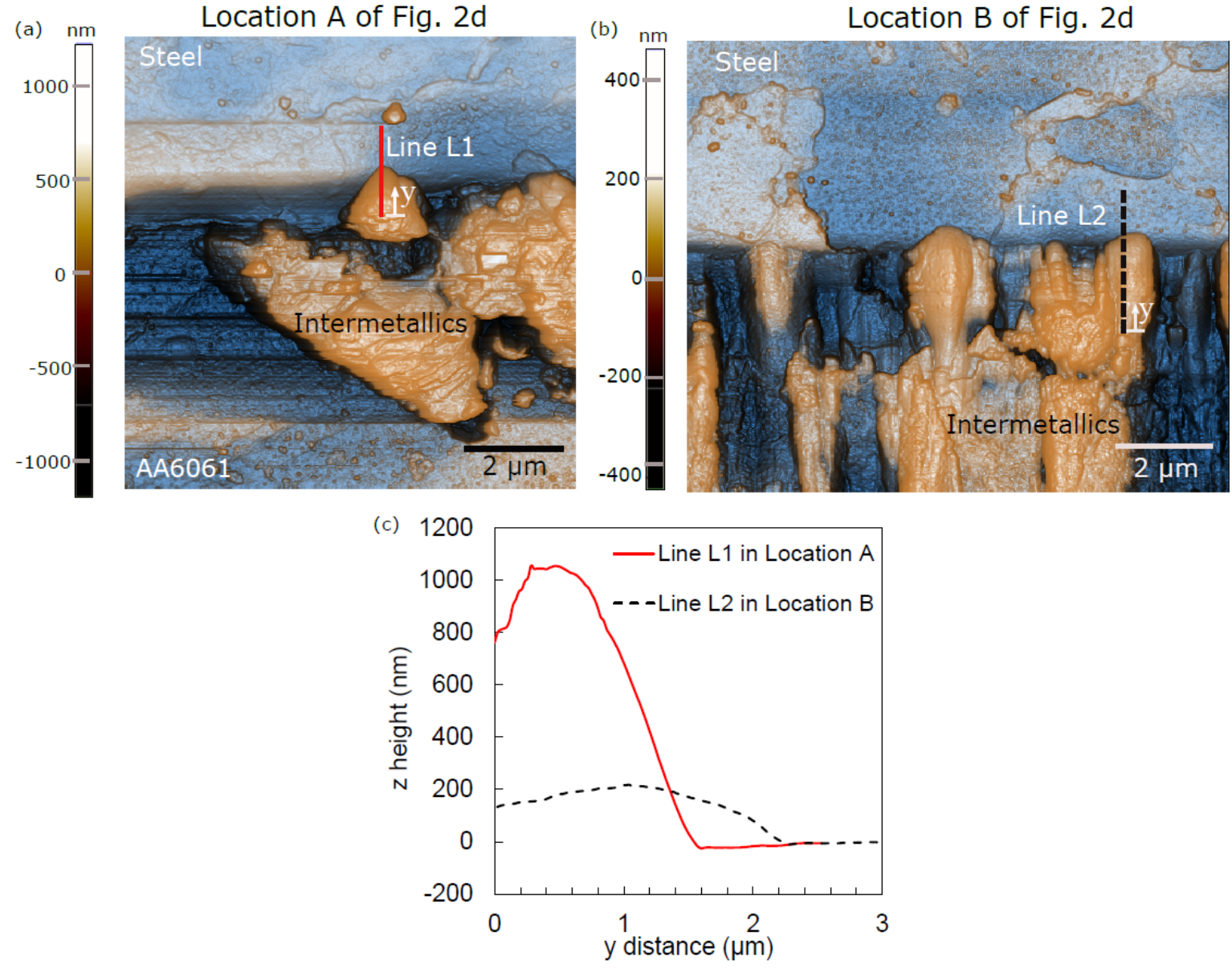

Fig. 3. AFM topography images showing the 3D morphology of the IMs at the Al/Fe interface: (a) IM nucleation (at location A of Fig. 2d) and (b) further directional IM growth across the interface (at location B of Fig. 2d) [color legends indicate the z- height]. (c) Surface profiles corresponding to line L1 (marked in Fig. 3a) and line L2 (marked in Fig. 3b) across the interface. Additional 3D views of the AFM maps are provided in Fig. S2 in the Supplementary Material. 
For accurate identification of the formed IM compounds, the same selected interface zones (delimited by a yellow rectangle in Fig. 2d, including locations A-F) were investigated by EBSD phase map, see Fig. 4a. IPF maps of the interface in the $x, y, z$ directions are provided in Fig. S3 in the Supplementary Material. It is worth noting that some variations in morphology are expected due to sample surface preparation. Quantitative analysis of the phase map (Fig. 4a) shows that $79 \%$ of the IM compounds formed at the interface during heat treatment at $596{ }^{\circ} \mathrm{C}$ for $60 \mathrm{~s}$ correspond to the $\theta$-phase and the remaining $21 \%$ to the $\eta$-phase. This confirms that the $\theta$-phase is the 'major phase' in this in situ experiment. Moreover, some locations across the interface (marked by black arrows in Fig. 4a) appear fully covered only by the $\theta$-phase, confirming that the $\theta$-phase nucleates first. In some regions, the $n$ phase also appears at the interface (aqua blue zone in Fig. 4a, e.g. zone B, C and E), confirming that its formation occurs at a later stage. This scenario is also in good agreement with the outcomes of the AFM investigation, where the zone corresponding to location A (Fig. 3a) was assumed to contain only the $\theta$-phase, while location B (Fig. 3b) contains both $\theta$ - and $\eta$ - phases. These observations clearly demonstrate that the terms 'major phase' and/or 'minor phase' are not suitable for the early stages of IMs nucleation and growth. It depends on the stage of growth that the IM has reached and might even be location dependent.

Histograms of grain size distribution for both $\theta$ - and $\eta$-phases are provided as supplementary in Fig. S4. Both phases are characterized by ultra-fine structure. The earlier formed $\theta$ - grains are slightly larger with an average size of $0.22 \pm 0.09 \mu \mathrm{m}$ compared to the $\eta$-grains of $0.20 \pm 0.09 \mu \mathrm{m}$. Both $\theta$ - and $\eta$-grains are slightly elongated along the phase growth direction, presenting very similar average aspect ratios of 1.84 and 1.86 , respectively. It should be noted that these values are much lower compared to those measured on columnar grains of both phases in the well-grown IM layer with the thickness of 38-40 $\mu \mathrm{m}[26,27]$. As shown in Fig. $4 \mathrm{~b}$ the vast majority of $\eta$-grains have a strong $<001>$ texture (maximum intensity of 13.3) along the IM growth direction (i.e. perpendicular to the interface), as their growth is controlled by interdiffusion of atoms in that direction. In comparison, the $\theta$-phase controlled by the reaction rate has a weak random texture with a maximum intensity of 3.6. 


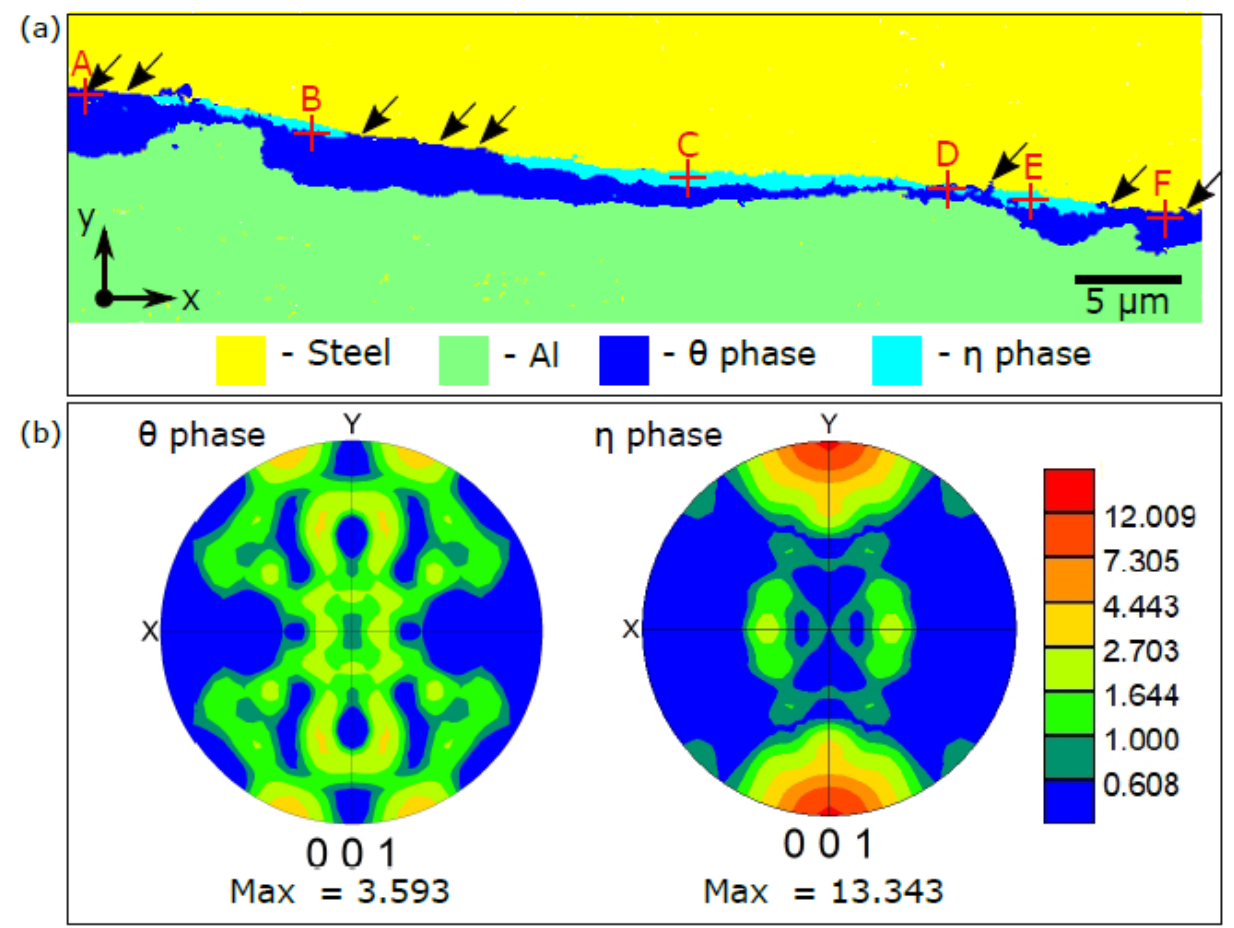

Fig. 4. (a) Phase map of the nucleated IM phases $(\theta$ and $\eta$ ) AA6061 and bcc-Fe from EBSD analysis after in situ heat treatment corresponding to the area enclosed by the yellow rectangle in Fig. $2 \mathrm{~d}$. (b) The $<001>$ texture for both IM phases. [IPF maps of the interface and grain size distributions are given in the Supplementary Material].

To sum up, this is the first-time that a dedicated high temperature in situ heating setup is used to successfully capture the onset of nucleation and growth of IMs at the Fe-Al interface. The in situ observations enabled to directly observe the location of initiation and to measure the growth rate of IMs. The sample could then be tracked from one technique to the next by location tracking. AFM confirmed that the nucleation and growth of IMs are associated with volume expansion as they are protruding out of plane. EBSD analysis revealed that, in some interface regions, only the $\theta$-phase is observed without emergence of the $\eta$ - phase. Coupling with the zone tracking identifying nucleation locations of the in situ SEM sample, it confirmed that the $\theta$-phase nucleates first. It is concluded that the terms minor and major - phases are not relevant for $\theta$ - and $\eta$ - phases at the early stages of their formation and growth. Indeed, the presence of different IMs depend on the growth time at high temperature, that can vastly change the observations. 


\section{Acknowledgements}

TS acknowledges F.R.S.-FNRS, (Belgium) for his postdoctoral fellowship at UCLouvain and for the research stay at IMDEA Materials Institute (Getafe, Spain). PX acknowledges gratefully the financial support from China Scholarship Council (No. 201606890031, Beijing, China). AS acknowledges the financial support of the European Research Council for a starting grant under grant agreement 716678, ALUFIX project. JMM-A acknowledges MAT4.0-CM project funded by Madrid region under program S2018/NMT-4381.

\section{References}

1. Raabe, D., J. Keichel, and G. Gottstein, Investigation of crystallographic slip in polycrystalline Fe3Al using slip trace measurement and microtexture determination. Acta Materialia, 1997. 45(7): p. 2839-2849.

2. $\mathrm{Li}, \mathrm{X}$. , et al., The Al-rich part of the Fe-Al phase diagram. Journal of Phase Equilibria and Diffusion, 2016. 37(2): p. 162-173.

3. Stein, F., et al., Determination of the crystal structure of the $\varepsilon$ phase in the Fe-Al system by high-temperature neutron diffraction. Intermetallics, 2010. 18(1): p. 150-156.

4. Chumak, I., K.W. Richter, and H. Ehrenberg, Redetermination of iron dialuminide, FeAl2. Acta Crystallographica Section C: Crystal Structure Communications, 2010. 66(9): p. i87-i88.

5. Scherf, A., et al., Orientation relationship of eutectoid FeAl and FeAl2. Journal of applied crystallography, 2016. 49(2): p. 442-449.

6. Matysik, P., S. Jóźwiak, and T. Czujko, Characterization of low-symmetry structures from phase equilibrium of Fe-Al system-Microstructures and mechanical properties. Materials, 2015. 8(3): p. 914-931.

7. Sun, Y., et al., Formation and phase transformation of aluminide coating prepared by lowtemperature aluminizing process. Surface and Coatings Technology, 2017. 330: p. 234-240.

8. van der Rest, C., P.J. Jacques, and A. Simar, On the joining of steel and aluminium by means of a new friction melt bonding process. Scripta Materialia, 2014. 77: p. 25-28.

9. Heumann, T. and N. Dittrich, Structure character of the Fe2Al5 intermetallics compound in hot dip aluminizing process. Z Metallk, 1959. 50: p. 617-623.

10. van der Rest, C., et al., On the reactive sintering of Heusler Fe2VAl-based thermoelectric compounds. Intermetallics, 2020. 125: p. 106890.

11. Richards, R., et al., Metallurgy of continuous hot dip aluminizing. International materials reviews, 1994. 39(5): p. 191-212.

12. Massalski, T.B., et al., Binary alloy phase diagrams. 1990, Ohio, USA: ASM International.

13. Shahverdi, H.R., et al., Microstructural analysis of interfacial reaction between molten aluminium and solid iron. Journal of Materials Processing Technology, 2002. 124(3): p. 345352.

14. Shahverdi, H., et al., Kinetics of interfacial reaction between solid iron and molten aluminium. Journal of Materials Science, 2002. 37(5): p. 1061-1066.

15. Bouayad, A., et al., Kinetic interactions between solid iron and molten aluminium. Materials Science and Engineering: A, 2003. 363(1): p. 53-61.

16. Springer, H., et al., On the formation and growth of intermetallic phases during interdiffusion between low-carbon steel and aluminum alloys. Acta Materialia, 2011. 59(4): p. 1586-1600. 
17. Hibino, A., Estimation of kinetic parameters for combustion synthesis of FeAl intermetallic compound by dipping experiment of Fe wire into Al melt. Materials transactions, 2010. 51(3): p. 516-524.

18. Farahani, H., et al., In Situ High-Temperature EBSD and 3D Phase Field Studies of the Austenite-Ferrite Transformation in a Medium Mn Steel. Microscopy and Microanalysis, 2019. 25(3): p. 639-655.

19. Zijlstra, G., et al., Interphase boundary motion elucidated through in-situ high temperature electron back-scatter diffraction. Materials \& Design, 2017. 132: p. 138-147.

20. Pasche, G., et al., Time-resolved X-ray microtomography observation of intermetallic formation between solid Fe and liquid Al. Metallurgical and Materials Transactions A, 2013. 44(9): p. 4119-4123.

21. Ding, Z., et al., Microstructural evolution and growth behavior of intermetallic compounds at the liquid Al/solid Fe interface by synchrotron X-ray radiography. Materials Characterization, 2018. 136: p. 157-164.

22. Novak, P., et al., On the formation of intermetallics in Fe-Al system-An in situ XRD study. Intermetallics, 2013. 32: p. 127-136.

23. Szczepaniak, A., Investigation of intermetallic layer formation in dependence of process parameters during the thermal joining of aluminium with steel. 2016, Universitätsbibliothek der RWTH Aachen: Aachen.

24. Govindaraj, N.V., J.G. Frydendahl, and B. Holmedal, Layer continuity in accumulative roll bonding of dissimilar material combinations. Materials \& Design (1980-2015), 2013. 52: p. 905-915.

25. Gedevanishvili, S. and S.C. Deevi, Processing of iron aluminides by pressureless sintering through $\mathrm{Fe}+\mathrm{Al}$ elemental route. Materials Science and Engineering: A, 2002. 325(1): p. 163176.

26. Sapanathan, T., et al., A new physical simulation tool to predict the interface of dissimilar aluminum to steel welds performed by friction melt bonding. Journal of Materials Science \& Technology, 2019. 35(9): p. 2048-2057.

27. Takata, N., et al., Crystallography of Fe2Al5 phase at the interface between solid Fe and liquid Al. Intermetallics, 2015. 67: p. 1-11. 